\title{
Gene network analysis and design
}

\author{
Matthew J. Berryman ${ }^{a}$, Andrew Allison ${ }^{a}$ and Derek Abbott $^{a}$ \\ ${ }^{a}$ Centre for Biomedical Engineering and \\ School of Electrical and Electronic Engineering, \\ The University of Adelaide, SA 5005, Australia.
}

\begin{abstract}
Gene networks are composed of many different interacting genes and gene products (RNAs and proteins). They can be thought of as switching regions in $n$-dimensional space or as mass-balanced signaling networks. Both approaches allow for describing gene networks with the limited quantitative or even qualitative data available. We show how these approaches can be used in modeling the apoptosis gene network that has a vital role in tumor development. The open question is whether engineering changes to this network could be used as a possible cancer treatment.
\end{abstract}

Keywords: gene networks, signaling, apoptosis, cancer

\section{INTRODUCTION}

While cancer is understood in very broad terms ${ }^{1,2}$ and many of the key proteins involved are very well understood, ${ }^{3-5}$ more research is needed at the gene network level to understand interactions between genes, proteins, and the environment. p53 is a key protein involved in a number of key cell processes, ${ }^{6}$ which if disturbed can lead to cancer. ${ }^{1,2}$ By modeling the p53 gene network, we can gain a better understanding of its interactions, with key implications for cancer treatment.

Modeling gene networks gives us a broad, yet important view of vital cell regulatory functions. ${ }^{7,8}$ Two techniques that have been developed are mass-balanced signaling networks ${ }^{9-11}$ (of which the Michaelis-Menten kinetics are a particular form ${ }^{12}$ ), and piecewise-linear differential equations (DEs), ${ }^{7,13,14}$ a generalization on the work in Boolean $N K$ switching networks by Glass and Edwards. ${ }^{14-16}$ Although enzyme kinematics are a useful tool, ${ }^{9,12}$ the ODEs involved are typically quite stiff which increases the computation time. ${ }^{10}$ Also, the piecewise-linear DE approach gives a better picture of the qualitative nature of the behaviour, ${ }^{7}$ since they are quick and accurate to integrate ${ }^{14}$ and good analytical tools exist for their qualitative behaviour. ${ }^{13}$

Gene networks present several problems for any sort of quantitative analysis. ${ }^{7,13}$ Many of the interacting proteins may be unknown, and even between the known interacting proteins, some of the interactions occur only under unknown conditions. ${ }^{7}$ Furthermore, although techniques such as microarrays and reverse transcriptasepolymerase chain reaction (RT-PCR) give gene expression levels, ${ }^{17}$ there is a lot of variance, a lot of measurement error, and they do not typically provide enough detail about the complex interactions to establish any strict rules of behaviour in the gene networks. ${ }^{18}$ Even in well-established gene networks, such as the p53 network, the details of many interactions are still being discovered. ${ }^{6}$ Some of the key ones, such as the feedback loop that exists between p53 and another protein, Mdm2, are lacking clear quantitative values. ${ }^{6}$ Consequently, we are left with an incomplete picture of protein interactions, with imprecise, qualitative rules - for example, protein A binds to protein $\mathrm{B}$, or protein $\mathrm{C}$ activates transcription of protein D. Basing our analysis on logical switching systems is useful, because:

"logical switching systems capture major features of a homologous class of nonlinear dynamical systems governed by sigmoidal functions because such systems tend to sharpen their responses to extremal values of the variables." (Kauffman $\left.{ }^{8}\right)$

Send correspondence to Derek Abbott

E-mail: dabbott@eleceng.adelaide.edu.au, Telephone: +61 883035748 
If one considers a typical sigmoidal function,

$$
y=\frac{x^{n}}{\theta^{n}+x^{n}},
$$

then it is trivial to show that

$$
\lim _{n \rightarrow \infty} y=\lim _{n \rightarrow \infty} \frac{\frac{x^{n}}{\theta_{n}}}{1+\frac{x^{n}}{\theta^{n}}}= \begin{cases}1, & x>\theta \\ 0, & x<\theta .\end{cases}
$$

Thus in the extreme case, as $n \rightarrow \infty$ we have a simple switching, or step function. Piecewise-linear (PL) differential equation (DE) models, originally developed by Glass and Edwards, ${ }^{14-16}$ offer a way around this problem, in that they can successfully model systems in a qualitative sense, including quantitative data where available. Filippov successfully introduced step-functions, which are important for modeling the switch-like regulatory interactions in gene networks. ${ }^{19}$

\section{METHODS}

As described by de Jong, ${ }^{13}$ the rates of change of proteins can be written as

$$
\dot{\mathbf{x}}=\mathbf{f}(\mathbf{x})-\mathbf{g}(\mathbf{x}) \mathbf{x}
$$

where $\mathbf{x}=\left(x_{1}, \ldots, x_{n}\right)^{\prime}, \mathbf{x} \in \mathbb{R}_{\geq 0}^{n}$ is the expression levels (copy number / volume) of the $n$ proteins, $\mathbf{f}=$ $\left(f_{1}, \ldots, f_{n}\right)^{\prime}$ consists of the rates of synthesis of each proteins, and $\mathbf{g}(\mathbf{x})=\operatorname{diag}\left(g_{1}, \ldots, g_{2}\right)$ is the rate of degradation. ${ }^{13}$ The rate of synthesis of the protein $i, f_{i}: \mathbb{R}_{\geq 0} \rightarrow\{0,1\}$ depends on the levels of the proteins, $\mathbf{x}$ in some fashion,

$$
f_{i}(\mathbf{x})=\sum_{l \in \mathcal{L}} \kappa_{i l} b_{i l}(\mathbf{x}),
$$

for rate parameter $\kappa_{i l}>0$, and $b_{i l}: \mathbb{R}_{>0}^{n} \rightarrow\{0,1\}$ a regulation function, and $\mathcal{L}$ a possibly empty set of indices of regulation functions. The $g_{i}$ are defined similarly to the $f_{i}$, except we require that $g_{i}>0 \forall i$. The regulation functions $b_{i l}$ are defined in terms of step functions $\mathrm{s}^{+}, \mathrm{s}^{-}: \mathbb{R}^{2} \rightarrow\{0,1\}$ defined as

$$
\begin{aligned}
& \mathrm{s}^{+}\left(x_{j}, \theta_{j}\right)= \begin{cases}1, & x_{j}>\theta_{j} \\
0, & x_{j}<\theta_{j}\end{cases} \\
& \mathrm{s}^{-}\left(x_{j}, \theta_{j}\right)=1-\mathrm{s}^{+}\left(x_{j}, \theta_{j}\right)
\end{aligned}
$$

We consider a generalized view of the p53 network, as shown in Figure 1. Based on Figure 1, we abstract over the proteins apart from p53 and Mdm2, instead considering $\mathbf{x}=\left(x_{d}, x_{u}, x_{p}, x_{m}, x_{A}, x_{C}, x_{D}\right)^{\prime}$ where $x_{d}$ is the level of DNA damage kinases, $x_{u}$ the level of UV stress kinases, $x_{p}$ the expression level of p53, $x_{m}$ the level of $\operatorname{Mdm} 2, x_{D}$ the combined level of apoptosis (programmed cell death) proteins, $x_{A}$ for the angiogenesis proteins, and $x_{C}$ for the cell cycle proteins. The network of interactions is shown in Figure 2. We use the following set of equations to model the p53 network:

$$
\begin{aligned}
x_{p} & =s_{p d} \mathrm{~s}^{+}\left(x_{d}, \theta_{d}\right)+s_{p u} \mathrm{~s}^{+}\left(x_{u}, \theta_{u}\right)+s_{p m} \mathrm{~s}^{+}\left(x_{m}, \theta_{m}\right) \mathrm{s}^{-}\left(x_{p}, \theta_{p}\right)-g_{p} x_{p} \\
x_{m} & =s_{m} \mathrm{~s}^{+}\left(x_{m}, \theta_{m}\right) \mathrm{s}^{-}\left(x_{p}, \theta_{p}\right)-g_{m} x_{m} \\
x_{A} & =s_{A} \mathrm{~s}^{+}\left(x_{p}, \theta_{A}\right)-g_{A} x_{A} \\
x_{C} & =s_{C} \mathrm{~s}^{+}\left(x_{p}, \theta_{C}\right)-g_{C} x_{C} \\
x_{D} & =s_{D} \mathrm{~s}^{+}\left(x_{p}, \theta_{D}\right)-g_{D} x_{D},
\end{aligned}
$$

where $\theta_{a}$ for some protein (or protein group) $a$ is the level at which the protein $b$ in the term $s_{c b} \mathrm{~s}^{ \pm}\left(x_{c}, \theta_{a}\right)$ signals for the synthesis of the protein $c$ at rate $s_{c b}$. No equations for $x_{d}$ and $x_{u}$ are given as these are input variables. We define $\theta_{C}<\theta_{A}<\theta_{D}$, which has the interpretation that as a first response the cell stops the cell cycle and 


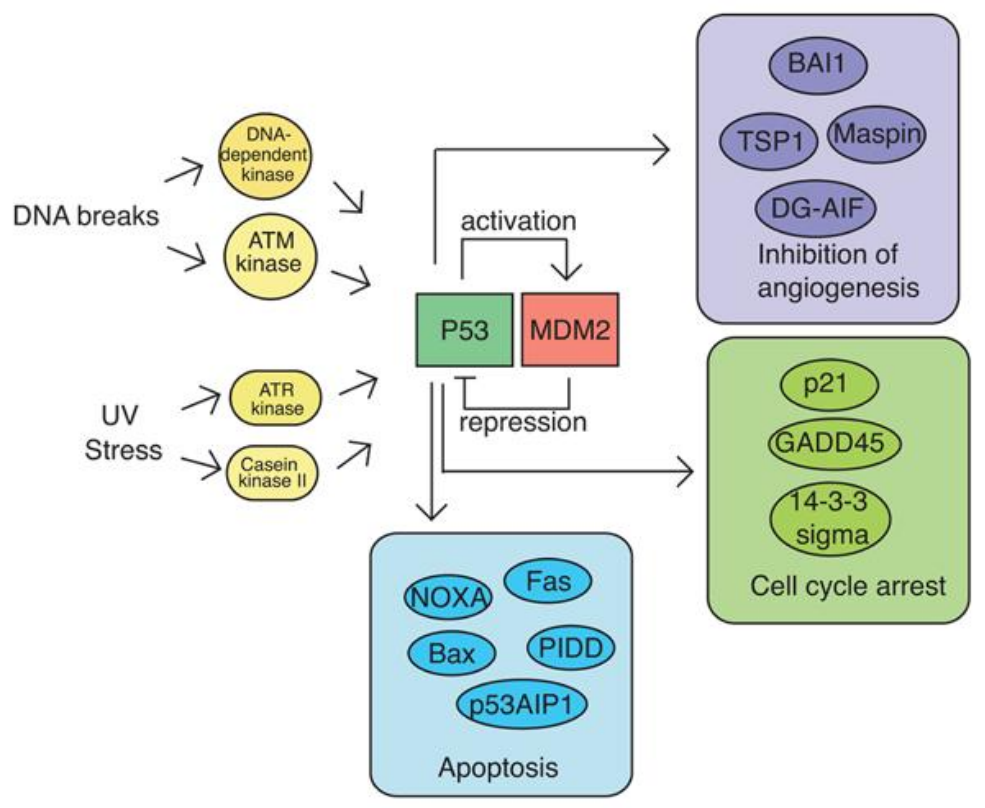

Figure 1. This figure, by Nakade, ${ }^{20}$ shows some of the key players in the p53 network, including the p53-Mdm2 feedback loop. DNA breaks and UV stress cause changes in such kinase proteins as ATM kinase and Casein kinase II. A kinase is a protein that adds a phosphor atom to a protein. In this case, a phosphor atom is added to p53. p53 is then termed phosphorylated, and this affects its binding to $\mathrm{Mdm} 2$, altering the feedback loop. The increased levels of p53 then can signal for inhibition of angiogenesis, cell cycle arrest (to allow DNA repair to occur), and/or apoptosis. The actual response, simply halting or undergoing apoptosis, is a function of the input.

signals for angiogenisis, and with the highest levels of damage and stress undergoes apoptosis (programmed cell death).

For each output variable, we have a target equilibrium position, which exists in some regulatory domain. Switching domains exist on the boundaries between regulatory domains. This can be seen in the representation of the domains for p53 and Mdm2 in Figure 3.

The equilibrium positions and the domains are shown in Table 1 . Note that $m_{a}$ is the maximum value of expression of protein $a$, denoted by $x_{a}$. For the output protein levels of interest, we also define critical values if the protein level $x_{A}$ lies above the critical value $c_{A}$, then we say protein group $A$ is activated, that is the cell would then be signaling for angiogenesis. These are the primary domains used for our analysis, however we have an alternate set to see what happens if we can reduce the level of p53 produced in response to various stress levels. These are shown in Table 2. We also need a set of initial conditions. Because this is a qualitative model, it is sufficient to simply specify regulatory domains for the initial conditions. We are interested in four different cases,

1. $\left(x_{d}, x_{u}\right) \in\left[0, \theta_{d}\right) \times\left[0, \theta_{u}\right)$,

2. $\left(x_{d}, x_{u}\right) \in\left[0, \theta_{d}\right) \times\left(\theta_{u}, m_{u}\right]$,

3. $\left(x_{d}, x_{u}\right) \in\left(\theta_{d}, m_{d}\right] \times\left[0, \theta_{u}\right]$, and

4. $\left(x_{d}, x_{u}\right) \in\left(\theta_{d}, m_{d}\right] \times\left(\theta_{u}, m_{u}\right]$,

corresponding to varying stresses on the cell; the first one corresponding to no DNA damage or UV stress. In addition, we must also specify the initial conditions for the other variables, these are kept constant as shown in Table 3.

$x \in(a, b)$ means $a<x<b, x \in(a, b]$ means $a<x \leq b, x \in[a, b)$ means $a \leq x<b$ and $x \in[a, b]$ means $a \leq x \leq b$ 


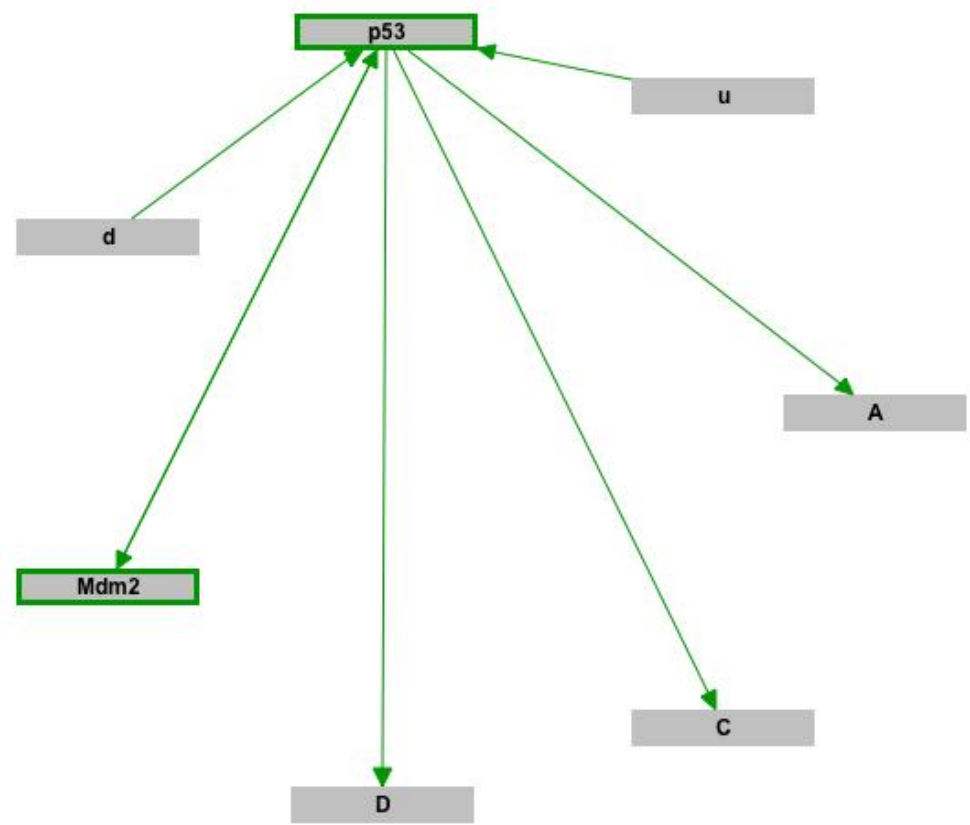

Figure 2. This network shows with directed arrows the influences of various inputs and proteins on other proteins. UV stress (denoted by $\mathrm{u}$ ) and DNA damage (d) signal for production of p53, which is in a feedback loop with the Mdm2 protein. Downstream targets of p53 include proteins that signal for angiogeneis (a), halting of the cell cycle (c), and apoptosis (programmed cell death, denoted by d).

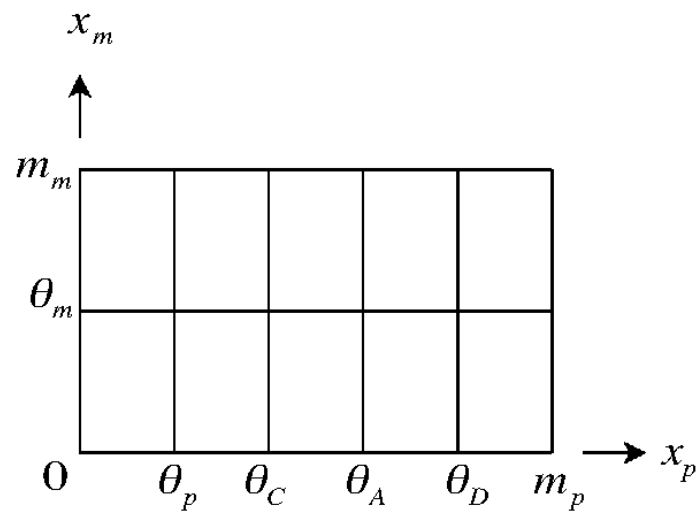

Figure 3. The set of protein expression values $\left(x_{p}, x_{m}\right) \in\left[0, m_{p}\right] \times\left[0, m_{m}\right]$ can be drawn as a $2 \mathrm{D}$ plane, with points lying either in switching domains, the lines at critical values $(\theta$ 's), and the regulatory domains (lying between critical values). As the level of p53 $\left(x_{p}\right)$ increases, it shifts in to different signaling domains. For example if $x_{p}$ lies in the range $\left(\theta_{C}, \theta_{A}\right)$ it means that cell-cycle halting is being signaled for, but not angiogenesis or apoptosis. 
Table 1. The equilibrium position(s) for each output protein level, and the regulatory domains that have been specified for these to occur in. There are several p53 equilibrium positions - one for each combination of synthesis/binding rates, the others have only one synthesis rate so only one equilibrium position occurs, according to the theorems in de Jong. ${ }^{13}$

\begin{tabular}{|c|c|c|}
\hline Variable & Equilibrium position & Regulatory Domain \\
\hline \hline$x_{p}$ & $\left(s_{p d}+s_{p u}\right) / g_{p}$ & $\left(\theta_{D}, m_{m}\right]$ \\
\hline$x_{p}$ & $\left(s_{p d}+s_{p u}+s_{p m}\right) / g_{p}$ & $\left(\theta_{D}, m_{m}\right]$ \\
\hline$x_{p}$ & $s_{p d} / g_{p}$ & $\left(\theta_{A}, \theta_{D}\right)$ \\
\hline$x_{p}$ & $\left(s_{p d}+s_{p m}\right) / g_{p}$ & $\left(\theta_{D}, m_{m}\right]$ \\
\hline$x_{p}$ & $s_{p u} / g_{p}$ & $\left(\theta_{A}, \theta_{D}\right)$ \\
\hline$x_{p}$ & $\left(s_{p u}+s_{p m}\right) / g_{p}$ & $\left(\theta_{A}, \theta_{m}\right)$ \\
\hline$x_{p}$ & $s_{p m} / g_{p}$ & $\left(\theta_{m}, m_{m}\right]$ \\
\hline \hline$x_{m}$ & $s_{m} / g_{m}$ & $\left(c_{C}, m_{C}\right]$ \\
\hline \hline$x_{C}$ & $s_{C} / g_{C}$ & $\left(c_{A}, m_{A}\right]$ \\
\hline \hline$x_{A}$ & $s_{A} / g_{A}$ & $\left(c_{D}, m_{D}\right]$ \\
\hline \hline$x_{D}$ & $s_{D} / g_{D}$ & \\
\hline
\end{tabular}

Table 2. Similar to Table 1, we list the lowered equilibrium position(s) for each output protein level, and the regulatory domains that have been specified for these to occur in. As discussed previously, p53 has several equilibrium positions - one for each combination of synthesis/binding rates, the others have only one synthesis rate so only one equilibrium position occurs, according to the theorems in de Jong. ${ }^{13}$

\begin{tabular}{|c|c|c|}
\hline Variable & Equilibrium position & Regulatory Domain \\
\hline \hline$x_{p}$ & $\left(s_{p d}+s_{p u}\right) / g_{p}$ & $\left(\theta_{A}, \theta_{D}\right)$ \\
\hline$x_{p}$ & $\left(s_{p d}+s_{p u}+s_{p m}\right) / g_{p}$ & $\left(\theta_{D}, m_{m}\right]$ \\
\hline$x_{p}$ & $s_{p d} / g_{p}$ & $\left(\theta_{C}, \theta_{A}\right)$ \\
\hline$x_{p}$ & $\left(s_{p d}+s_{p m}\right) / g_{p}$ & $\left(\theta_{A}, \theta_{D}\right)$ \\
\hline$x_{p}$ & $s_{p u} / g_{p}$ & $\left(\theta_{C}, \theta_{A}\right)$ \\
\hline$x_{p}$ & $\left(s_{p u}+s_{p m}\right) / g_{p}$ & $\left(\theta_{A}, \theta_{D}\right)$ \\
\hline$x_{p}$ & $s_{p m} / g_{p}$ & $\left(\theta_{C}, \theta_{A}\right)$ \\
\hline \hline$x_{m}$ & $s_{m} / g_{m}$ & $\left(\theta_{m}, m_{m}\right]$ \\
\hline \hline$x_{C}$ & $s_{C} / g_{C}$ & $\left(c_{A}, m_{A}\right]$ \\
\hline \hline$x_{A}$ & $s_{A} / g_{A}$ & $\left(c_{D}, m_{D}\right]$ \\
\hline \hline$x_{D}$ & $s_{D} / g_{D}$ & \\
\hline
\end{tabular}

Table 3. Initial regulatory domains for p53 $\left(x_{p}\right)$, Mdm2 $\left(x_{m}\right)$, and output signals for the halting of cell cycle $\left(x_{C}\right)$, angiogenesis $\left(x_{A}\right)$, and apoptosis $\left(x_{D}\right)$.

\begin{tabular}{|c|c|}
\hline Variable & Initial domain \\
\hline \hline$x_{p}$ & {$\left[0, \theta_{A}\right]$} \\
\hline$x_{m}$ & {$\left[0, m_{m}\right]$} \\
\hline$x_{C}$ & {$\left[0, c_{C}\right]$} \\
\hline$x_{A}$ & {$\left[0, c_{A}\right]$} \\
\hline$x_{D}$ & {$\left[0, c_{D}\right]$} \\
\hline
\end{tabular}




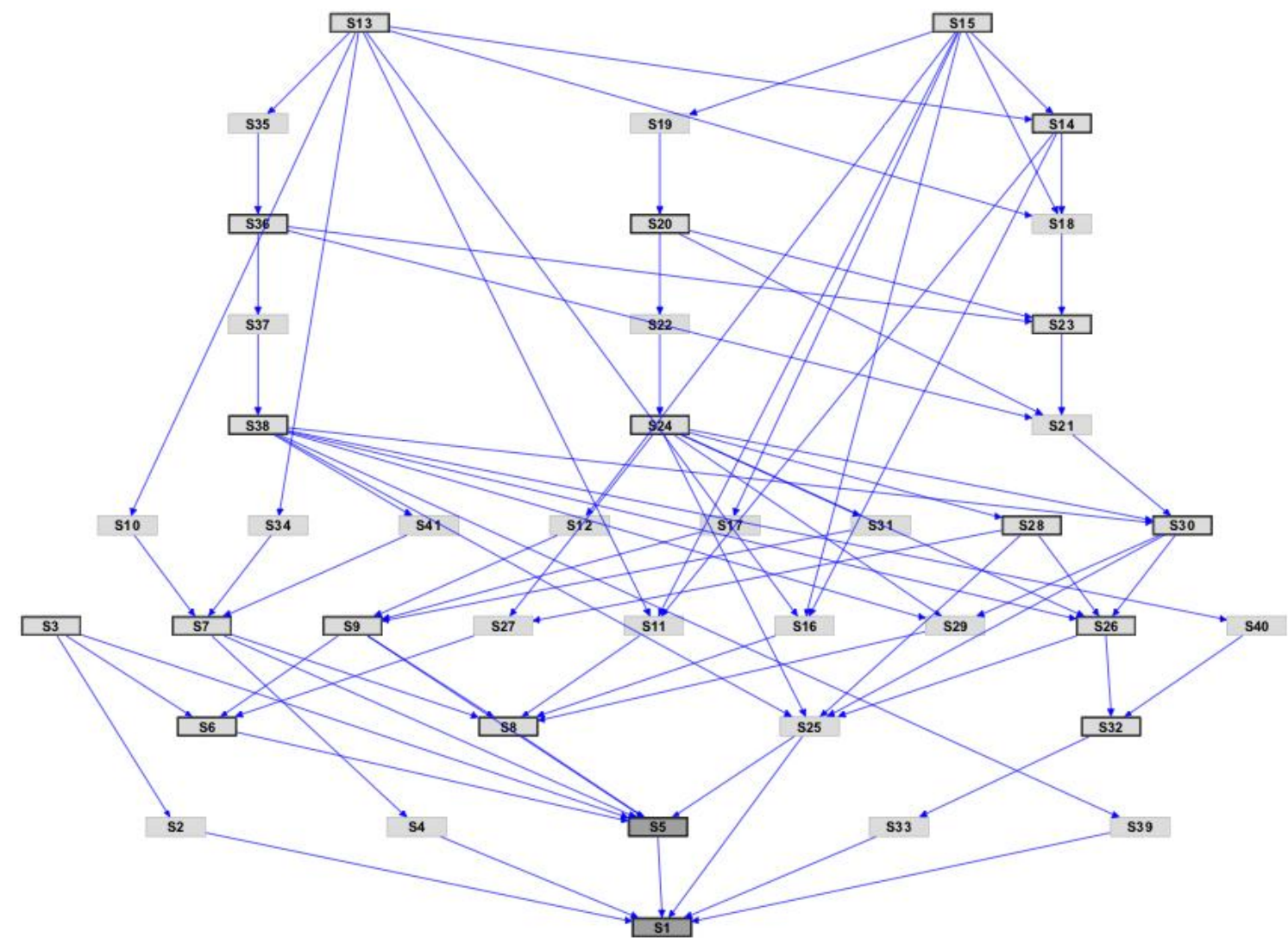

Figure 4. Graph showing the final set of states for the input set one, $\left(x_{d}, x_{u}\right) \in\left[0, \theta_{d}\right) \times\left[0, \theta_{u}\right)$, representing no external stress on the cell. States denote the proteins being in particular domains, for example Tables 4 denotes the final state S1. The numbering of states is arbitrary. From the initial equilibrium state, the cell can transition through a number of regulatory (boxed) and switching (not boxed) states and lying in an attractor basis with the final equilibrium state in Table 4 as the attractor. Arrow directions denote allowed state transitions.

\section{RESULTS}

Each set of initial conditions run through the model produces a set of states that the cell cycles through. We found that in each case there is a single attractor state that the system settles into, representing the decision of the cell to either continue normally, halt the cell cycle, signal for angiogenesis, or undergo apoptosis. An example of the transition diagram is shown in Figure 4. The set of four states, corresponding to the final states for each input 1-4, and the normal equilibrium postions in Table 1 are shown in Tables 4 through 7.

Table 4. Final state for input set one: $\left(x_{d}, x_{u}\right) \in\left[0, \theta_{d}\right) \times\left[0, \theta_{u}\right)$. This represents no external stress on the cell. As expected, none of its responses are triggered, represented by $x_{a}<c_{A}, x_{C}<c_{C}$ and $x_{D}<c_{D}$.

\begin{tabular}{|c|c|}
\hline Variable & Final domain \\
\hline \hline$x_{A}$ & {$\left[0, c_{A}\right)$} \\
\hline$x_{C}$ & {$\left[0, c_{C}\right)$} \\
\hline$x_{D}$ & {$\left[0, c_{D}\right)$} \\
\hline$x_{m}$ & {$\left[0, \theta_{m}\right)$} \\
\hline$x_{p}$ & {$\left[0, \theta_{p}\right)$} \\
\hline
\end{tabular}

For the alternate set of equilibrium positions in Table 2, the results are the same, except that when a single input is present, the system only signals for the cell cycle to be halted and not angiogensis, and making $c_{A}<c_{C}$ 
Table 5. Final state for input set two: $\left(x_{d}, x_{u}\right) \in\left[0, \theta_{d}\right) \times\left(\theta_{u}, m_{u}\right]$. This represents one external stress on the cell, in this case UV stress. Two of its responses are triggered, represented by $c_{A}<x_{a} \leq m_{A}, c_{C}<x_{C} \leq m_{C}$ and $c_{D}<x_{D} \leq m_{D}$. In words, this means the cell is signaling for angiogenesis, and has halted the cell cycle in order to prevent DNA damage, but the cell is not under enough pressure to undergo apoptosis.

\begin{tabular}{|c|c|}
\hline Variable & Final domain \\
\hline \hline$x_{A}$ & $\left(c_{A}, m_{A}\right]$ \\
\hline$x_{C}$ & $\left(c_{C}, m_{C}\right]$ \\
\hline$x_{D}$ & {$\left[0, c_{D}\right)$} \\
\hline$x_{m}$ & $\theta_{m}$ \\
\hline$x_{p}$ & $\left(\theta_{A}, \theta_{D}\right)$ \\
\hline
\end{tabular}

Table 6. Final state for input set three: $\left(x_{d}, x_{u}\right) \in\left(\theta_{d}, m_{d}\right] \times\left[0, \theta_{u}\right]$. This represents one external stress on the cell, in this case DNA damage. Two of its responses are triggered, represented by $c_{A}<x_{a} \leq m_{A}, c_{C}<x_{C} \leq m_{C}$ and $c_{D}<x_{D} \leq m_{D}$. In words, this means the cell is signaling for angiogenesis, and has halted the cell cycle to repair DNA damage, but the cell is not under enough pressure to undergo apoptosis.

\begin{tabular}{|c|c|}
\hline Variable & Final domain \\
\hline \hline$x_{A}$ & $\left(c_{A}, m_{A}\right]$ \\
\hline$x_{C}$ & $\left(c_{C}, m_{C}\right]$ \\
\hline$x_{D}$ & {$\left[0, c_{D}\right)$} \\
\hline$x_{m}$ & $\theta_{m}$ \\
\hline$x_{p}$ & $\left(\theta_{A}, \theta_{D}\right)$ \\
\hline
\end{tabular}

Table 7. Final state for input set four: $\left(x_{d}, x_{u}\right) \in\left(\theta_{d}, m_{d}\right] \times\left(\theta_{u}, m_{u}\right]$. This represents one external stress on the cell, in this case DNA damage. Two of its responses are triggered, represented by $c_{A}<x_{a} \leq m_{A}, c_{C}<x_{C} \leq m_{C}$ and $c_{D}<x_{D} \leq m_{D}$. In words, this means the cell is so stressed and damaged that it would be too risky for cell division to occur, which would fix in the genetic changes to its descendants, so it undergoes apoptosis.

\begin{tabular}{|c|c|}
\hline Variable & Final domain \\
\hline \hline$x_{A}$ & $\left(c_{A}, m_{A}\right]$ \\
\hline$x_{C}$ & $\left(c_{C}, m_{C}\right]$ \\
\hline$x_{D}$ & {$\left[c_{D}, m_{D}\right)$} \\
\hline$x_{m}$ & $\theta_{m}$ \\
\hline$x_{p}$ & $\left(\theta_{A} \theta_{D}\right)$ \\
\hline
\end{tabular}

reverses that. Also for both inputs being present, the output is that both the cell cycle and angiogenesis are signaled for, but not apoptosis. This allows potentially harmful mutations to be passed on to daughter cells.

\section{CONCLUSIONS}

Given a qualitative model of the interactions of the p53 protein in the cell, we are able to design a simplified gene network to represent this information. In a qualitative sense this gives the output that is observed in in vivo and in vitro cells, namely that if not stressed or damaged, the cell undergoes a normal cell cycle and divides. If there is some damage or stress the cell cycle is halted, and for a significant amount of damage and stress the cell does not divide and pass on its mutations but instead undergoes apoptosis (programmed cell death). Shifting the equilibrium positions is equivalent to altering the response of the cell; for the example we considered, changing the equilibrium positions lower means the cell is less likely to signal for appropriate responses, in particular never undergoing apoptosis. This would mean that potentially harmful (to the organism) mutations are carried forward into daughter cells upon division of the stressed and damaged cell. Shifting the equilibrium positions higher means the cell is more likely to halt and repair DNA and undergo apoptosis for higher (yet lower than normal) levels of DNA damage. This could be a useful response to have in tumors that have not lost the capability of apoptosis and suggests future treatments could target these equilibrium positions.

In future work, we propose exploring the angiogenesis, cell cycle, and apoptosis pathways in more detail. 
The use of quantitative data where available would allow us to build testable models of cell outputs, although our existing model agrees with observed qualitative descriptions of cell processes. In addition to exploring more equilibrium positions and inputs, we can also explore changes to the network. Since many drugs alter the pathways, we could possibly impact upon drug selection, dosage, and development of new drugs for treating cancer.

\section{Acknowledgments}

We appreciate the useful discussions with Katherine Gordon (Flinders University, Australia) and Sabrina Spencer (Massachusetts Institute of Technology, USA) in this work. We acknowledge funding from The University of Adelaide.

\section{REFERENCES}

1. S. L. Spencer, M. J. Berryman, J. García, and D. Abbott, "An ordinary differential equation model for the multistep transformation to cancer," Journal of Theoretical Biology 231, pp. 515-524, 2004.

2. D. Hanahan and R. Weinberg, "The hallmarks of cancer," Cell 100, pp. 57-70, 2000.

3. P. A. Futreal, L. Coin, M. Marshall, T. Down, T. Hubbard, R. Wooster, N. Rahman, and M. R. Stratton, "A census of human cancer genes," Nature Reviews Cancer 4, pp. 177-183, 2004.

4. J. W. Arends, "Molecular interactions in the Vogelstein model of colorectal carcinoma," Journal of Pathology 190, pp. 412-416, 2000.

5. A. G. Knudson, "Cancer genetics," American Journal of Medical Genetics 111, pp. 96-102, 2002.

6. B. Vogelstein, D. Lane, and A. J. Levine, "Surfing the p53 network," Nature 408, pp. 307-310, 2000.

7. H. de Jong, J. Geiselmann, G. Batt, C. Hernandez, and M. Page, "Qualitative simulation of the initiation of sporulation in bacillus subtilis," Bulletin of Mathematical Biology 66, pp. 261-299, 2004.

8. S. A. Kauffman, The Origins of Order: Self-Organization and Selection in Evolution, Oxford University Press, 1993.

9. B. Aleman-Meza, Y. Yu, H.-B. Schüttler, J. Arnold, and T. R. Taha, "KINSOLVER: A simulator for compting large ensembles of biochemical and gene regulatory networks," in http://lsdis.cs.uga.edu/ aleman/kinsolver/, 2004.

10. H. Kurata, T. Inoue, Y. Sumida, S. Tanaka, and T. Ohashi, "Simulation and system analysis of gene regulatory networks using the two-phase partition method," Genomes Informatics 12, pp. $286-287,2001$.

11. M. Kærn, W. J. Blake, and J. J. Collins, "The engineering of gene regulatory networks," Ann. Rev. Biomed. Eng. 5, pp. 179-206, 2003.

12. J. D. Murray, Mathematical Biology, Springer, 3rd ed., 2002.

13. H. de Jong, J.-L. Gouzé, C. Hernandez, M. Page, T. Sari, and J. Geiselmann, "Quantitative simulation of genetic regulatory networks using piecewise-linear models," Bulletin of Mathematical Biology 66, pp. 301340, 2004.

14. R. Edwards and L. Glass, "Combinatorial explosion in model gene networks," Chaos 10, pp. 691-704, 2000.

15. L. Glass, "Classification of biological networks by their qualitative dynamics," Journal of Theoretical Biology 54, pp. 85-107, 1975.

16. R. Edwards, "Analysis of continuous-time switching networks," Physica D 146, pp. 165-199, 2000.

17. D. D. Shoemaker, E. E. Schadt, C. D. Armour, Y. D. He, P. Garrett-Engele, P. D. McDonagh, L. P. M., et al., "Experimental annotation of the human genome using microarray technology," Nature 409, pp. 922-927, Feb. 2001

18. Y. Moreau, F. de Smet, G. Thijs, K. Marchal, and B. de Moor, "Functional bioinformatics of microarray data: from expression to regulation," Proceedings of the IEEE 90, pp. 1722-1743, Nov. 2002.

19. A. F. Filippov, Differential equations with discontinuous righthand sides, Kluwer Academic Publishers, 1988.

20. K. Nakade, "The p53 network," in http://www.brc.riken.jp/lab/dna/en/GENESETBANK/p53_network.html, 2004. 